New Concepts in Imaging: Optical and Statistical Models

D. Mary, C. Theys and C. Aime (eds)

EAS Publications Series, 59 (2013) 189-201

\title{
IMAGING TECHNIQUES IN MILLIMETRE ASTRONOMY
}

\author{
M. Bremer ${ }^{1}$
}

\begin{abstract}
Compared to optical astronomy, millimetre radio astronomy experiences not only a different and complementary aspect of the universe but also different perturbations and limitations from Earth's atmosphere that are mostly imposed by atmospheric water vapour and its dynamics. After discussing the physics behind the refractive index variations and possible correction schemes, a small introduction into the basics of radio interferometry and image reconstruction with the CLEAN algorithm is given.
\end{abstract}

\section{Introduction}

Millimetre Astronomy is a powerful tool to observe the cold, molecular gas in space, ranging from nearby objects in the Solar system over targets in our Galaxy to galaxies so remote that their light has been travelling for more than $90 \%$ of the age of the universe before reaching Earth.

Radio interferometry differs in a number of important points from optical interferometry. Real and imaginary parts of the incoming signal are detected and correlated electronically, observations can be performed at daytime and night time with only weather-imposed limitations, and Earth's atmosphere becomes a bright background against which the astronomical source must be detected.

\section{Atmosphere}

In the optical, astronomical observations are perturbed by seeing: a large number of small $(\sim 10 \mathrm{~cm})$, rapid refractive index fluctuations in the atmosphere leads to multiple splitting of an image into speckles that change with a rate of about $100 \mathrm{~Hz}$ (Lohman et al. 1983). The influence of speckles diminishes in the near infrared, but so does the atmospheric transmission when moving further to longer wavelengths: Extended absorption line systems of mainly water vapour and carbon dioxide block ground-based astronomical observations. When observations

\footnotetext{
${ }^{1}$ Institut de Radio Astronomie Millimétrique (IRAM), 300 rue de la Piscine, 38406 Saint-Martin-d'Hères, France
} 


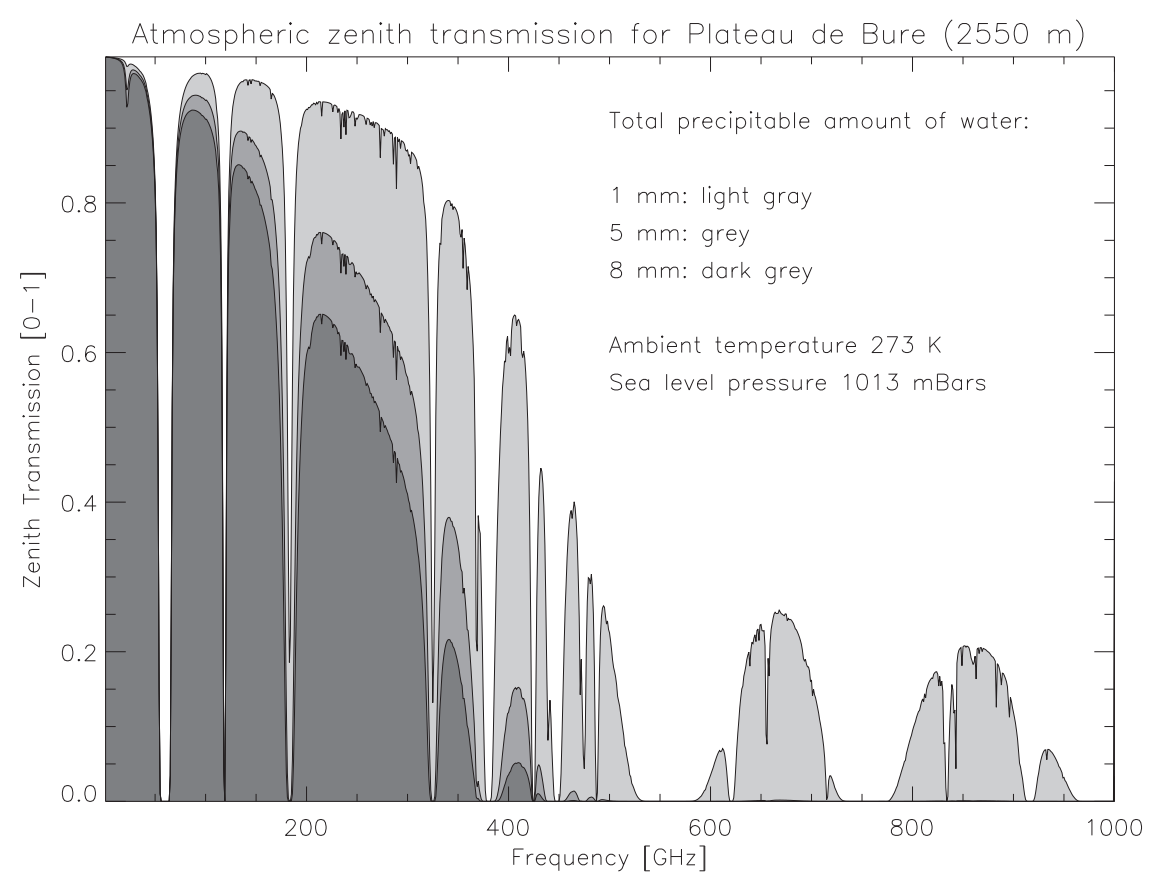

Fig. 1. Atmospheric transmission on Plateau de Bure, France calculated with the ATM model (Pardo et al. 2001) for different amounts of total precipitable water. Water vapour has an exponential scale height of only $2 \mathrm{~km}$ (dry air has $8.4 \mathrm{~km}$ ) allowing high altitude sites to extend the edge of the radio window to higher frequencies.

become possible again at the short wavelength edge of the radio window (see Fig. 1), seeing has completely changed its character. It is now slow $(0.1-0.01 \mathrm{~Hz})$ and moves the whole image around instead of splitting it into speckles. Obviously, radio waves become sensitive to an atmospheric component that does not influence optical wavelengths in the same way. This component is water vapour. Its extended absorption line systems between the optical and radio windows do more than locally absorb emission. For $\mathrm{H}_{2} \mathrm{O}$ a total of 114, 241, 164 spectral lines are recorded in the HITRAN V13.0 database (Rothman et al. 2009, the number of $\mathrm{H}_{2} \mathrm{O}$ transitions is given on the associated web pages); each line introduces a long-range step into the refractive index according to the Kramers-Kronig relation that links the real and imaginary parts of the dielectric constant. When solving the Kramers-Kronig relation for the whole spectrum is impractical, local approximations based on experimental measurements can be employed. Numerical atmospheric radiative transfer codes like ATM (Pardo et al. 2001, also available in the ASTRO software within the GILDAS-IRAM package ${ }^{2}$ ) or AM (Paine 2012) often use fitted pseudo-continua to approximate distant line wings for opacities,

\footnotetext{
${ }^{2}$ http://www.iram.fr/IRAMFR/GILDAS
} 


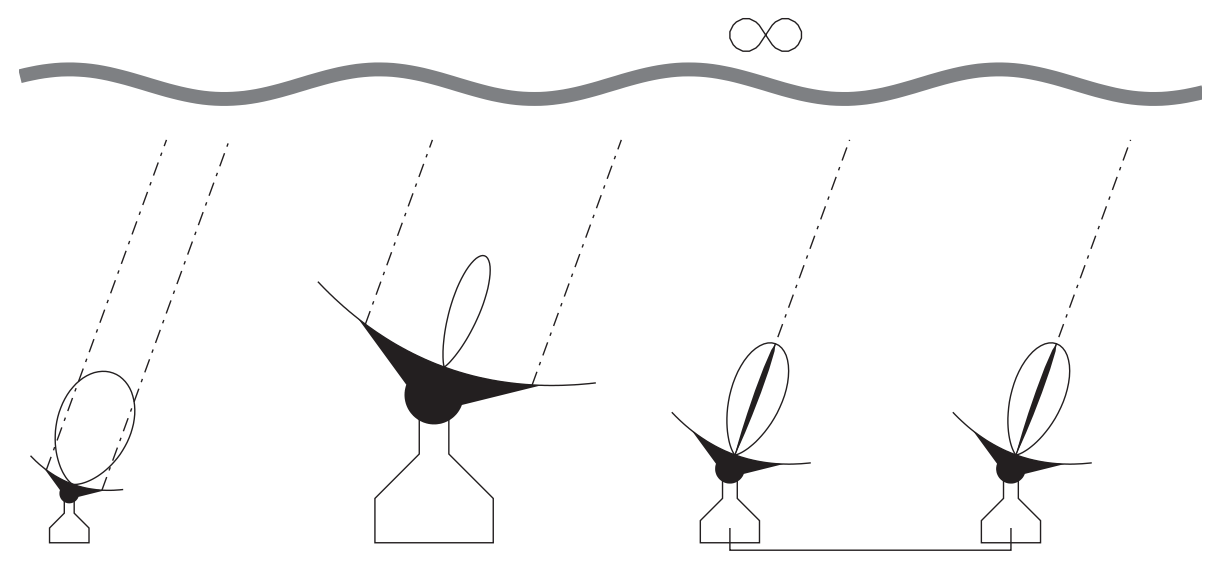

Fig. 2. Ground-based radio observatories observing the (nearly) infinite sky. At a given frequency, small antennas have a wide beam, large antennas a fine beam, and interferometers wide individual beams but narrow synthesised beams.

and may use an empirical step in the refractive index calculation when they reach the edge of their line database (15 THz for AM 7.2). For numerical values for the refractive index in the radio range (without using a model code) see e.g. Hill \& Cliffort (1981). The dependency of the total refractive index on ambient temperature, dry pressure and partial vapour pressure can be taken into account over the Smith - Weintraub equation (Smith \& Weintraub 1953, see also Thompson et al. 1986).

Water vapour has several unusual physical properties. Under terrestrial conditions it can change between its gaseous, liquid and solid states, act as an important heat transfer medium, is lighter than dry air but stays in the lower atmosphere due to the negative tropospheric temperature gradient. Other than most atmospheric gasses, water vapour mixes badly with the dry atmosphere and tends to form bubbles of some metres to kilometres in size. Those bubbles have a tendency to merge but are opposed in this by the action of turbulence, which tends to create a weather-dependent power law size distribution of the bubbles and their associated refractive index perturbations that levels off to a constant high level when the outer scale of turbulence is reached. This "phase screen" can move horizontally with the wind, making wind speed an important parameter for phase noise even when the total amount of precipitable water is low. The refractive index variations impact radio observations on two levels (Fig. 2). In single-dish telescopes, it is known as "anomalous refraction" (see Altenhoff et al. 1987; Downes \& Altenhoff 1990) and typically indicates that the single beam of a telescope seems to move around the requested position. Large radio telescopes have a finer beam resolution than small antennas at the same frequency, and are therefore more sensitive to this phenomenon. In extreme cases this can perturb observations so much that no meaningful data can be obtained: the beam can wander over neighbouring parts of 
an extended source, stay an unknown fraction of time off-source for an unresolved target, and can cause severe errors during the pointing and focus calibrations.

For interferometers with their extended baselines, phase variations can be noticed well before the beams of the individual antennas become perturbed (Fig. 3). Also for the synthesised beam, the source seems to wander around slowly (Fig. 4); but other than the single dish anomalous refraction that is sensitive to the total fluctuation of the water vapour column, the phase on a baseline is only sensitive to the difference of the fluctuations along the lines of sight of the connected antennas. This distinction is advantageous for compact interferometer configurations under summer conditions, when the outer scale of turbulence can be in the kilometre range (PdBI baseline lengths in compact 6Dq configuration are $24-97 \mathrm{~m}$ ).

An important impact of phase noise on the integrated amplitude is expressed in the formula $\left\|V_{\text {obs }}\right\|=\left\|V_{\text {real }}\right\| \cdot \exp \left(-\phi^{2} / 2\right)$ where $\phi$ is the phase noise in radians, $V_{\text {obs }}$ the observed integrated visibility, and $V_{\text {real }}$ the visibility in the absence of atmospheric phase noise. As the phase noise scales to good approximation linearly with observing frequency, observing conditions can be prohibitive for band 3 and 4 , marginal for band 2, and correct for band 1 (see Fig. 5 for the frequency ranges of the bands). Telescopes that operate in service mode can choose the project that is best adapted to meteorological conditions. But when only fixed telescope time slots are offered to observers, this flexibility does not exist.

Statistically, atmospheric parameters like temperature, wind speed and refractive index variations can be treated as non-stationary random processes. This means that not only individual measurement values but also their averages wander around in time. Classical averages and their variations are in such a situation ill defined. An elegant method to characterise those processes are structure functions, and the view of turbulence as an energy transport mechanism from large to small scales, where the continuously sub-dividing turbulent eddies become finally small enough to dissipate their kinetic energy as heat (Tatarski 1961; Kolmogorov 1941a,b, 1991a,b).

\section{Water vapour radiometry}

The most reliable way to correct for the phase noise generated by turbulent water vapour is real-time remote monitoring close to the observed line of sight, with a time resolution of the order of telescope diameter divided by typical wind speed. A radiometer is a receiver that is sensitive to the thermal emission of water vapour. In order to distinguish between clouds and gaseous vapour, radiometers are employed that monitor the contrast between the cloud-generated continuum and a water vapour line. The droplets in clouds contribute significantly to the detected emission, but only little to the optical path; their rejection is therefore essential for a meaningful phase correction in the presence of clouds. The increased opacity on a water vapour line causes a stronger coupling to the vertical temperature distribution in the atmosphere, and thus an increase in the power received by the radiometer. Figure 5 shows how precisely the atmospheric emission needs to be measured (in Kelvin) as a function of monitoring frequency to obtain a given 

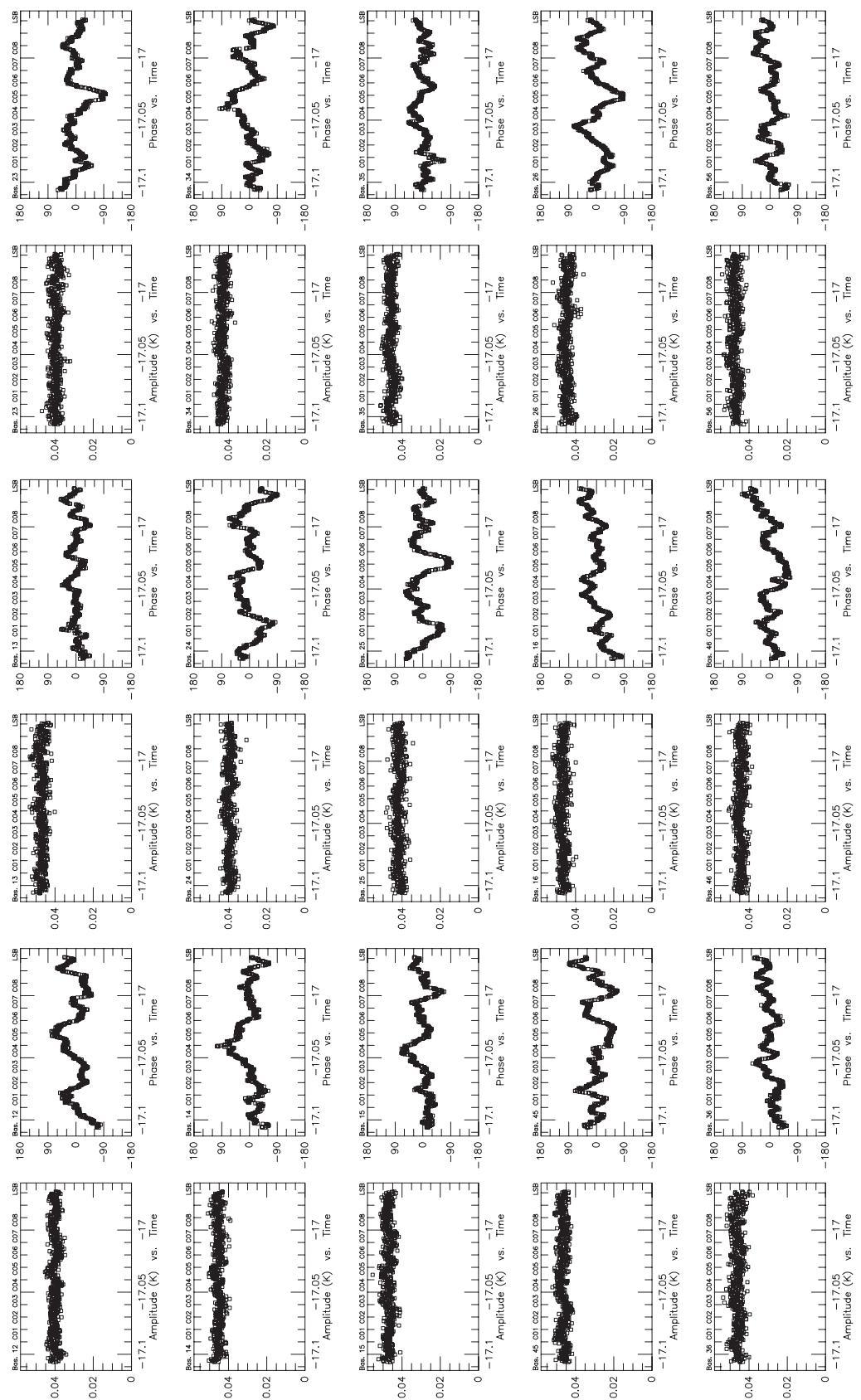

Fig. 3. Amplitudes and phases on a strong point source during $495 \mathrm{~s}$. The stability of the amplitudes shows that individual antenna beams do not wander around on the sky. The phases, however, move significantly. 

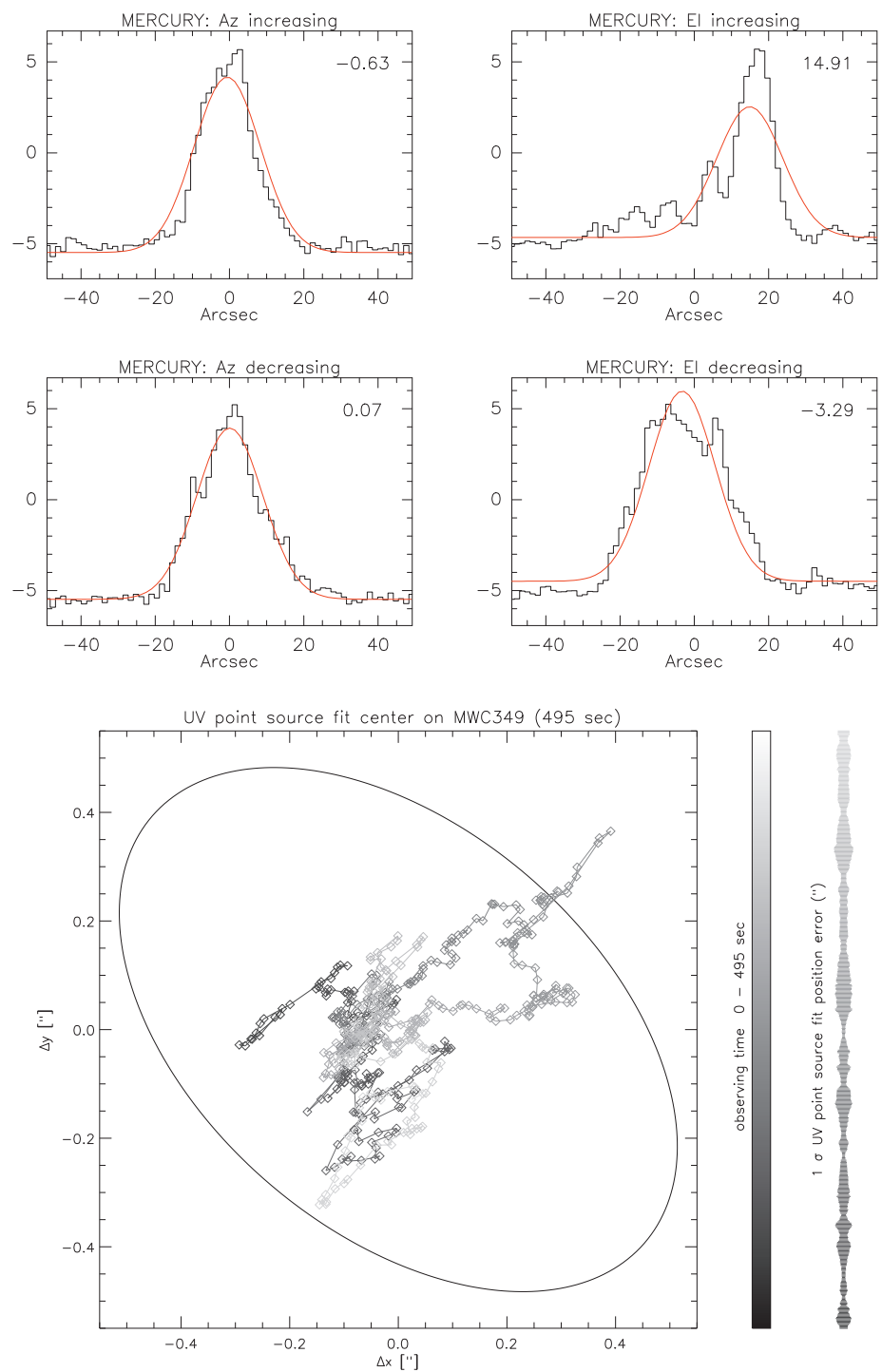

Fig. 4. Top: pointing scan at the IRAM 30-m telescope under conditions of anomalous refraction. The scan consists of two drifts in azimuth and elevation over Mercury. Bottom: the amplitudes and phases of Figure 3 expressed as the movement of MWC 349 (time sequence coded in grey levels) observed at $88.950 \mathrm{GHz}$. The 1-sigma error bar (rightmost vertical trace) as a function of time shows that the movement is significant. The ideal clean beam half-power contour is indicated by an ellipse.

path rms. Other criteria must also be taken into account: electronic components of high stability, low price and ambient temperature operation are available for 


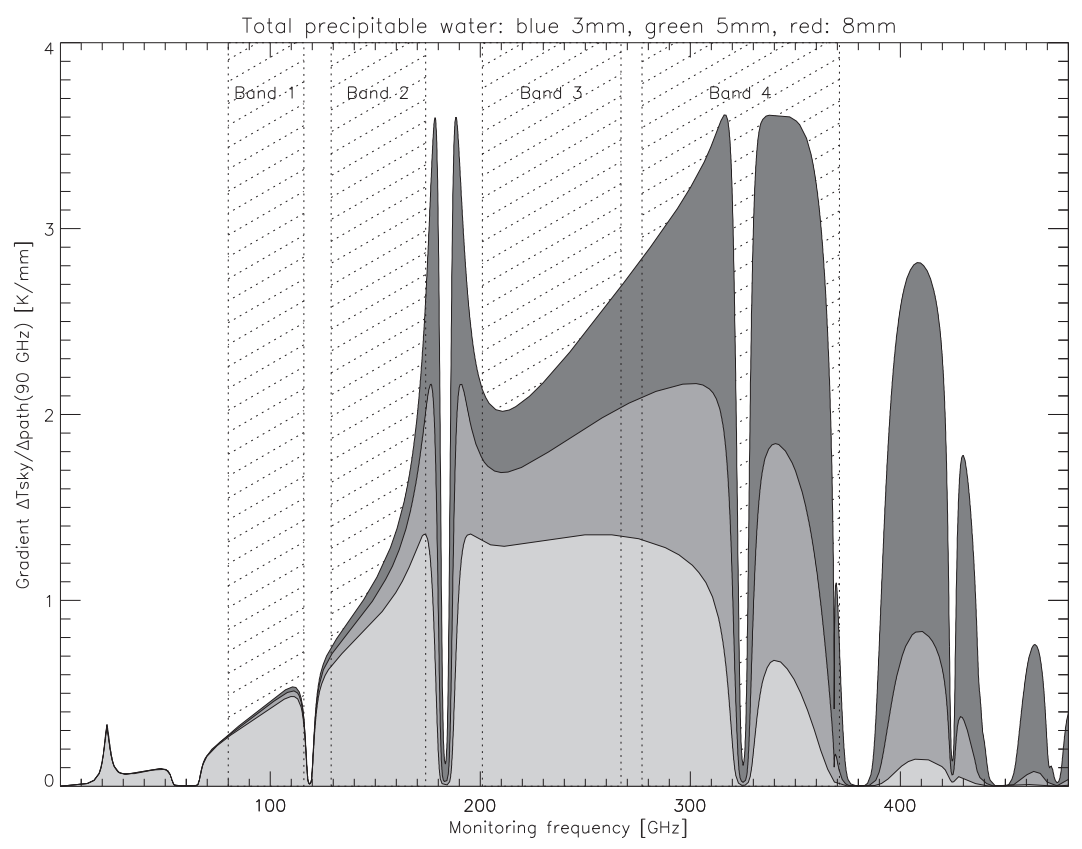

Fig. 5. Gradient $\Delta T_{\text {sky }} / \Delta$ path for $3 \mathrm{~mm}$ (dark grey) $5 \mathrm{~mm}$ (grey) and $8 \mathrm{~mm}$ (light grey) total precipitable water, calculated as a function of monitoring frequency for a fixed observing frequency of $90 \mathrm{GHz}$ at Plateau de Bure $(2550 \mathrm{~m})$. The observing bands 1-4 of the interferometer are indicated.

lower frequencies while components for high frequencies may require cooling, or need more elaborate calibration techniques to counter drifts in performance. The smaller opacity $\tau$ at frequencies lower than the astronomical receiver bands allows to measure the whole tropospheric line of sight under observing conditions, while high monitoring frequencies might reach their $\tau=1$ layer not far from the antennas and thus stay insensitive to an important fraction of the observing band phase fluctuations. And finally, interference by satellite emitters or telecommunication relays is more common at lower frequencies, with a tendency to rise over the years as main-stream technologies evolve and the requests for increased communication bandwidth become more pressing. Each observatory must therefore carefully choose the type of radiometer that is adapted to its needs. For the Plateau de Bure, the total power signal of the $1 \mathrm{~mm}$ astronomical receivers was used between 1995 and mid-2004, after mid-2004 the system was fully switched to dedicated $22 \mathrm{GHz}$ radiometers with cloud correction. These radiometers use three $1 \mathrm{GHz}$ large channels centred on $19.2 \mathrm{GHz}, 22.0 \mathrm{GHz}$ and $25.2 \mathrm{GHz}$ and operate at ambient temperature. Figure 6 illustrates the benefit of the radiometric phase correction: The phase noise is not only lower, but also less dependent on baseline length, which helps to conserve the angular resolution of the resulting map. In 2012, the radiometers on Bure were only perturbed by two satellite beacons, but 


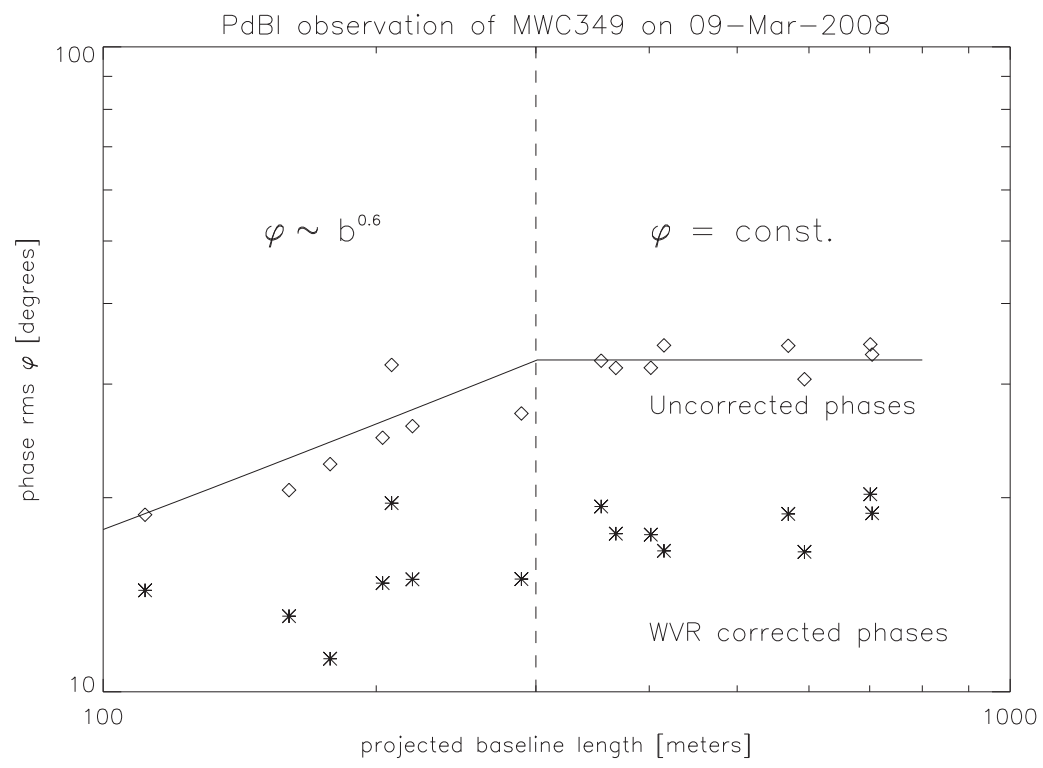

Fig. 6. Baseline-specific phase rms for the observations in Figure 3 versus projected baseline length, with and without $22 \mathrm{GHz}$ radiometric phase correction. The vertical dashed line indicates the approximate outer scale size of the turbulent water vapour cells on that day.

it required extended negotiations and the goodwill of several telecommunication operators to keep powerful ground-based signal relays in the $16-24 \mathrm{GHz}$ range away from the neighbourhood of the observatory.

\section{Image formation in radio interferometry}

Radio interferometry is one of the few domains in science where the Fourier transform of a desired quantity is observed, and needs to be inversely transformed (van Cittert-Zernike theorem, Fraunhofer diffraction). This subject has been treated in depth in many excellent textbooks and conference proceedings (e.g. Thompson et al. 1986; Perley et al. 1986; Taylor et al. 1989, 1999). Combining radio telescopes into an interferometer requires a number of basic conditions:

- The antennas must observe the source simultaneously.

- The detected signal is a complex visibility vector that can be expressed as an amplitude and phase, and which is baseline-specific. As a consequence, both antennas must share a common frequency reference against which the phase can be measured. This can be done by either distributing a signal from one master reference to all antennas (connected element interferometer), or to use frequency standards of sufficient stability on each antenna (e.g. very long baseline interferometry (VLBI)). 
- Individual antennas have their primary beam that corresponds to a single pixel detector, with a diffraction-limited resolution defined by the reflector diameter. However, within this primary beam each combination of two antennas can resolve details with a diffraction-limited resolution of the antenna spacing. As each combination of antennas counts, the number of simultaneous baselines is given by $N_{\text {bas }}=N_{\text {ant }} \cdot\left(N_{\text {ant }}-1\right) / 2$, i.e. adding one antenna to an array of five increases the number of simultaneous baselines by $50 \%$. As the astronomical source rises, culminates and sets, the apparent orientation and projected length of each baseline changes and thus traces a curved line in the Fourier plane, which is also called UV plane (Earth rotation synthesis).

- Antennas cannot be spaced closer than the average of their diameters, and that means that the baseline "zero" that defines the integral flux cannot be accessed. This is the so-called "zero spacing" problem.

The higher the observing frequency and the larger the observing bandwidth, the more demanding become the technical requirements for the receivers, the signal transport with its delay compensation, and the correlator that needs to process the full spectral bandwidth of all baselines. The WIDEX wide-band correlator currently in service on the Plateau de Bure has a capacity of 914 Tera-operations per second (M. Torres, priv. com.).

\section{Gridding and image restoration techniques}

During the construction of a local interferometer, some effort has to be invested into the planning of station positions (also called "pads") where a mobile antenna can clamp down, connect to the system and start observing (see e.g. Kogan 2000; Boone 2001 \& Cohanim et al. 2004). The Earth rotation synthesis tracks of each baseline need to fill the UV plane as efficiently as possible for a variety of source declinations, while the total cost for building and cabling the stations must stay within reasonable limits. Over a year an interferometer will undergo a limited number of configuration changes, with closer spaced antennas during turbulent summer conditions and extended spacing during stable and dry winter conditions. An observer can apply to observe his/her source in several configurations to study it in different spatial resolutions, and to combine the result into a single map.

The resulting UV data are not regularly sampled and have no measurements at the centre; also, they are limited in their maximum UV coverage. In order to use fast Fourier Transform (FFT) methods, the observations need to be interpolated to a regular grid. That is typically done by convolving the UV data with a gridding function; the smoothing effect of the convolution is welcome because the individual visibilities are noisy samples of a locally smooth distribution. In order to avoid information loss, the grid spacing needs to be at least Nyquist sampled, and preferentially by a factor of two finer (for a smoother gridded image). The grid extend must be at least two times larger than the useful field of view to avoid folding back part of the valuable image information onto itself. 
With the inverse Fourier transform, the image corresponding to the UV data can be obtained. This image is called "dirty image" for a good reason: It shows positive and negative structure, and an integral of zero. Clearly, this data needs further treatment before it can be interpreted scientifically. One possibility is the fitting of UV models to the visibilities; in this case a careful study of the fit residuals is necessary but the resulting fit parameters have well-defined errors. The alternative is image restoration: the image is deconvolved, but then convolved again with an appropriate elliptical Gaussian beam.

In order to perform the deconvolution step, the instrumental point spread function (PSF) needs to be known. In radio interferometry this is the Fourier transform of an ideal point source, seen through the UV tracks of the real observation and thus precisely known. It has the same inconveniences as the observed image (zero integral, positive and negative structure) and is therefore named "dirty beam". Numerous techniques exist to obtain a deconvolved solution.

The most popular algorithm for the deconvolution and restoration of interferometer maps is CLEAN (Högbom 1974). It decomposes the source into a number of point-like components; its steps can be summarised as follows:

1. Deconvolution: First, set the residuals map to the dirty map, reset the list of point source components to zero, and choose a loop gain $\gamma=0.1 \ldots 0.3$ that stays the same during the following steps.

2. Identify the maximum absolute value $\left\|I_{\max }\right\|$ in the residual map.

3. Add $\gamma \cdot I_{\max }$ and its position to the list of point source components.

4. Subtract $\gamma \cdot$ PSF at this position from the residual map.

5. Go back to point (2) until the convergence criterion is reached. This can be the maximum number of iteration, a relative noise level expressed in terms of the maximum of the map, or an absolute noise level.

6. Restoration: The obtained result needs to be convolved with a clean beam, which is defined by fitting an elliptical Gaussian to the central part of the PSF. On scales smaller than the clean beam the deconvolution may create artifacts (no free "super resolution"), and this convolution will remove them.

7. Add the residual map to the clean map. This step is essential to allow noise estimates, and allows to reduce the effect if the cleaning was not deep enough.

It must be clearly stated that the result will be ambiguous and won't have a welldefined error estimate, but it will be closer to the real source structure than the dirty image and therefore a better basis for scientific analysis.

An important feature is to allow negative point source components. They can either appear as intermediate steps in the iteration, or can be a real part of the source structure if there is an absorption against an extended background emission (which may have been suppressed due to the spatial filtering). It is typically a good idea to keep track of the cumulative flux of positive and negative point source 

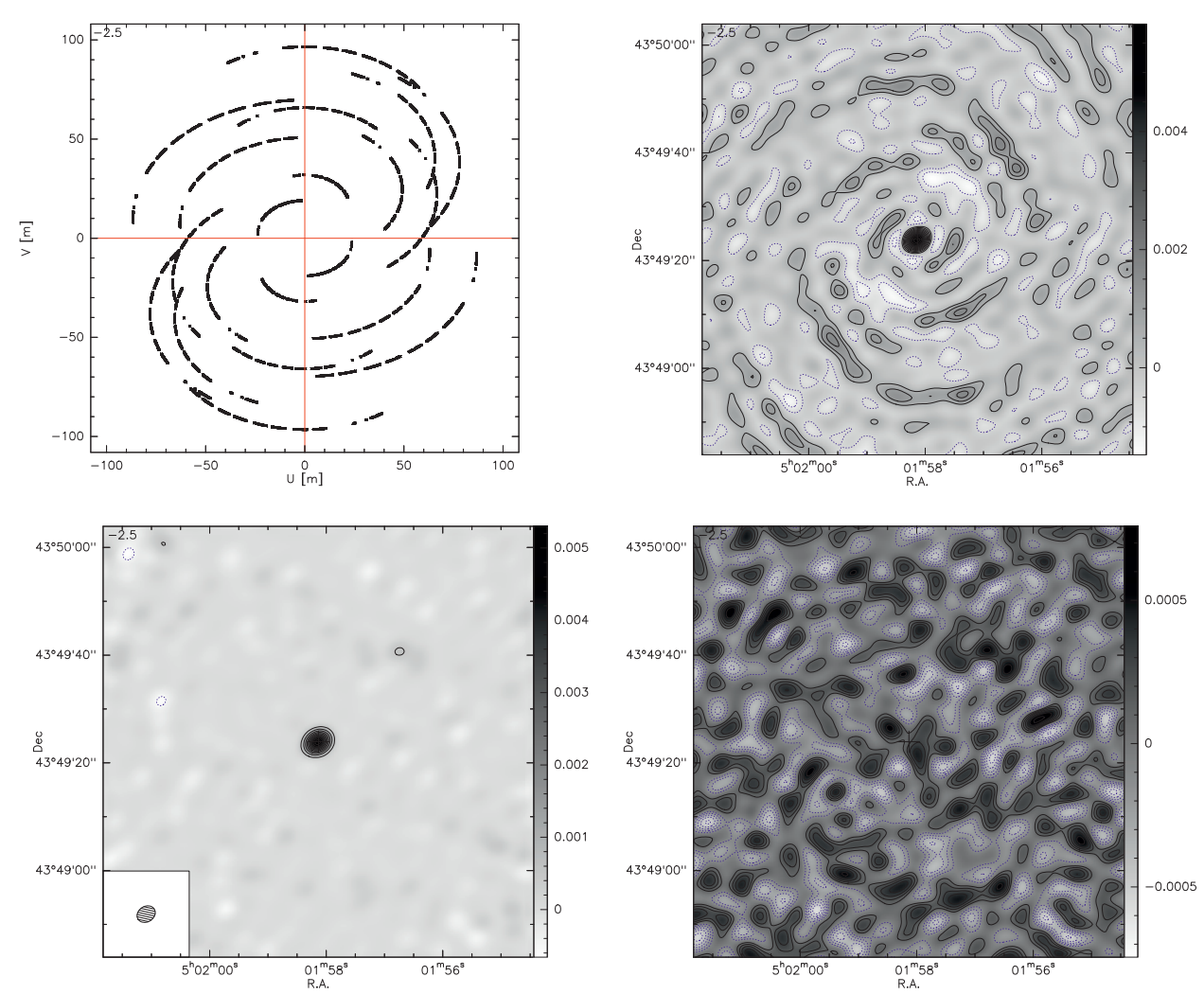

Fig. 7. Top row: UV coverage of a 5-antenna configuration, and the resulting dirty image of a point source. Bottom row: cleaned image (left) and residuals of a point source fit to the phase centre.

components during the iterations: if it reaches a stable level it is a good indicator that the deconvolution has converged. If it starts to oscillate strongly or diverges, the convergence criteria need to be adjusted.

It is possible to observe sources with an interferometer that are more extended than the primary beam of the individual antennas. In this case, the whole interferometer observes repeatedly a grid of pointings called "mosaic" on the sky (again, Nyquist sampling should be used). This technique allows to obtain several fields of view under comparable conditions. In this case it is necessary to obtain information on UV spacings shorter than the interferometer provides. This can be done with single dish observations and (if possible) with observations by an interferometer composed of smaller antennas, otherwise extended source structure will be missed. Already if the source is larger than $1 / 3$ of the primary telescope beam, its interferometer map starts to be affected. Fortunately IRAM operates not only an interferometer but also a large (30-m) single dish telescope that allows to obtain zero spacing observations in an uncomplicated way. 
Variants of CLEAN exist that address particular problems, sometimes in the context of overlapping multiple maps (Mosaics) or with an improved restoration of extended structure (see Schwab 1984; Steer et al. 1984; Wakker \& Schwarz 1988 and Cornwell 2008). Maximum entropy methods (see Richardson 1972; Lucy 1974; Narayan \& Nityananda 1986) are often not well adapted to cases where negative values are present in the dirty map. Their use may only be attempted if the dirty beam is nearly Gaussian.

\section{Conclusion}

Advances in signal transport, receiver and computer technology are important cornerstones of today's knowledge in millimetre astronomy. While the data acquisition and interpretation are less direct than for optical observations and require some experience, modern radio observatories provide an important support (pipeline-reduced data, local contact astronomers) for the scientists who want to use these tools. Over the Internet sites of many observatories (in the case of IRAM, http://www.iram-institute.org), astronomers can obtain the latest information on the further evolving instrumental capabilities and download dedicated data processing software. There still remain many secrets to be discovered beyond our planet, sometimes they are just one large step in observing frequency away.

The interferometric imaging, deconvolution and restoration sections of this article discuss material that was presented in much greater depth by J. Pety and F. Gueth in the context of the 7th IRAM Interferometry school (2010) (http://www.iram-institute.org/EN/content-page-2127-67-182-212-0.html).

\section{References}

Altenhoff, W.J., Baars, J.W.M., Wink, J.E., \& Downes, D., 1987, A\&A, 184, 381

Boone, F., 2001, A\&A, 377, 368

Cohanim, B.E., Hewitt, J.N., \& de Weck, O., 2004, ApJS, 154, 705

Cornwell, T.J., 2008, IEEE J. Selected Topics Signal Proc., 2, 793

Downes, D., \& Altenhoff, W.J., 1990, URSI/IAU Symposium on Radio Astronomical Seeing, 31

Hill, R.J., Cliffort, S.F., 1981, Radio Science, 16, 77

Högbom, J.A., 1974, A\&AS, 15, 417

Kogan, L., 2000, IEEE Trans. Antennas Propagation, 48, 1075

Kolmogorov, A.N., 1941a, Proc. USSR Acad. Sci., 30, 299 (Russian)

Kolmogorov, A.N., 1941b, Proc. USSR Acad. Sci., 32, 16 (Russian)

Kolmogorov, A.N., 1991a, Proceedings of the Royal Society of London, Series A: Math. Phys. Sci., 434, 9 (translated to English by V. Levin)

Kolmogorov, A.N., 1991b, Proceedings of the Royal Society of London, Series A: Math. Phys. Sci., 434, 15 (translated to English by V. Levin) 
Lohmann, A.W., Weigelt, G., \& Wirnitzer, B., 1983, Appl. Opt., 22, 4028

Lucy, L.B., 1974, AJ, 79, 745

Narayan, R., \& Nityananda, R., 1986, ARA\&A, 24, 127

Paine, S., 2012, SMA Technical Memo, No. 152

Pardo, J.R., Cernicharo, J., \& Serabyn, E., 2001, IEEE Trans. Antennas Propagation, 49, 1683

Perley, R.A., Schwab, F.R., Bridle, A.H., 1986, "Synthesis Imaging", NRAO Workshop, No. 13

Richardson, W.H., 1972, J. Opt. Soc. Am. (1917-1983), 62, 55

Rothman, L.S., Gordon, I.E., Barbe, A., et al., 2009, J. Quant. Spectrosc. Radiative Transfer, 110, 533 (web pages http://www.cfa.harvard.edu/hitran/)

Schwab, F.R., 1984, AJ, 89, 1076

Smith, E.K., \& Weintraub, S., 1953, Proc. IRE, 41, 1035

Steer, D.G., Dewdney, P.E., \& Ito, M.R., 1984, A\&A, 137, 159

Tatarski, V.I., 1961, "Wave propagation in a turbulent medium" (McGraw-Hill New York, Toronto, London)

Taylor, G.B., Carilli C.L., \& Perley, R.A., 1989, Astron. Soc. Pacific Conf. Ser., Vol. 6

Taylor, G.B., Carilli C.L., \& Perley, R.A., 1999, Astron. Soc. Pacific Conf. Ser., Vol. 180

Thompson, A.R., Moran, J.M., \& Swenson, G.W., 1986, "Interferometry and Synthesis in Radio Astronomy"

Wakker, B.P., \& Schwarz, U.J., 1988, A\&A, 200, 312 
